\title{
Estratégias fisioterapêuticas na prevenção da Pneumonia associada à Ventilação Mecânica
}

\author{
Physiotherapeutic strategies in the prevention of pneumonia \\ associated with mechanical ventilation
}

\author{
1 Fernanda Cortez Moraes \\ 2 Pâmela Camila Pereira \\ 3 Luís Henrique Sales Oliveira lhfisio@ig.com.br
}

$\begin{array}{ll}1 & \text { Fisioterapeuta Especialista em Fisioterapia Cardiorrespiratória - UGF. Docente da FEPI. } \\ 2 & \text { Fisioterapeuta Mestre em Reeducação do Equilíbrio Corporal - UNIAN. Docente da FEPI. } \\ 3 & \text { Fisioterapeuta Doutor em Ciências - UNIFESP. Docente da - FEPI. }\end{array}$

\section{RESUMO}

A Pneumonia Associada à Ventilação Mecânica (PAVM) é uma patologia de alta incidência e impacto significativo no tempo de internação, na morbidade e mortalidade dos pacientes e consequentemente nos custos para a instituição, por isso medidas de prevenção se mostram importantes. Este trabalho teve como objetivo revisar as principais estratégias da fisioterapia na prevenção da PAVM. Foram utilizados os estudos publicados que tiveram como referência as bases de dados Medline, Pubmed e Lilacs para selecionar os estudos de evidência científica no período entre 2000 e 2014 . O fisioterapeuta pode contribuir com a prevenção da patologia através de técnicas, gerenciamento e implantação de protocolos e programas de educação permanente para a equipe multiprofissional. A maioria dos estudos relatados na literatura considerou o efeito de apenas uma intervenção, o presente estudo propôs uma abordagem multinível, agrupando as medidas. Concluiu-se a fisioterapia tem ampla e ativa participação na adoção e no gerenciamento de medidas não farmacológicas para a prevenção da PAVM, que se destacam pela sua maior viabilidade, eficácia e considerável menor custo, contudo são necessárias mais pesquisas sobre $o$ assunto para incrementar as evidências sobre seus benefícios na literatura.

\section{Palavras-chave}

Pneumonia; respiração artificial; serviço hospitalar de fisioterapia; prevenção e controle.

\begin{abstract}
The Ventilator-Associated Pneumonia (VAP) is a disease of high incidence and significant impact on length of hospital stay, morbidity and mortality of patients and consequently, on cost to the institution, so preventive measures are of importance. This study aimed to analyze the key strategies of physical therapy in the prevention of VAP. We used studies published on Medline, Pubmed and Lilacs, of scientific evidence, in the period between 1996 and 2011. The physiotherapist may contribute to the prevention of disease through techniques, management and implementation of protocols and continuing education programs for a multidisciplinary team. Most studies reported in the literature only considered the effect of one intervention, whereas this study proposed a multilevel approach, grouping measures. The conclusion is that physiotherapy has broad and active participation in the adoption and management of nonpharmacological measures for the prevention of VAP, which stand out for their greater feasibility, efficacy and considerably lower cost; however, we need more research on the subject to increase the evidence in the literature about its benefits.
\end{abstract}

\section{Keywords}

Pneumonia; artificial respiration; hospital physiotherapy service; prevention and control.

\section{Como você deve citar?}

MORAES, Fernanda Cortez; PEREIRA, Pâmela Camila; OLIVEIRA, Luís Henrique Sales. Estratégias fisioterapêuticas na prevenção da Pneumonia associada à Ventilação Mecânica. Cadernos UniFOA, Volta Redonda, n. 31, p. 123-130, ago. 2016. 


\section{INTRODUÇÃO}

A pneumonia é definida como uma resposta inflamatória decorrente da penetração e multiplicação de microorganismos no trato respiratório inferior, enquanto a PAVM é definida como o a infecção do trato respiratório que se desenvolve no período de 48 horas após o início de ventilação mecânica ou no período de 48 a 72 horas após a extubação (ALMEIDA, POMBO, RODRIGUES, 2010).

Segundo Ricard (2006) apesar dos inúmeros avanços sobre essa pneumonia, seus indicadores continuam muito impactantes, com incidência é de 9 a $27 \%$ e mortalidade de 25 a $50 \%$, além de ser responsável pelo prolongamento no tempo de ventilação mecânica na UTI.

Para Craven e Hjalmarson (2010) a patologia complica o curso de 8 a $28 \%$ dos pacientes que receberam ventilação mecânica, esses pacientes parecem ter um risco de morte de 2 a 10 vezes maior em comparação com pacientes sem pneumonia.

A tecnologia altamente especializada e complexa utilizada em UTI favorece a sobrevida dos pacientes críticos, mas em contrapartida aumenta os fatores de riscos predisponentes para a infecção hospitalar. Já que os pacientes críticos necessitam de monitorização e suporte contínuo para preservação de suas funções vitais, e a maioria absoluta deles é submetida a procedimentos invasivos que prejudicam os mecanismos de defesa do trato respiratório, tendo como consequência a PAVM (ALMEIDA, POMBO, RODRIGUES, 2010; AZEVEDO, 2006).

A intubação orotraqueal é de fato o fator de risco determinante para o desenvolvimento da PAVM, uma vez que isoladamente aumenta de 6 a 20 vezes o risco, principalmente nos primeiros dias pós-intubação e diminui progressivamente a ser mínima após 2 semanas, segundo a American Thoracic Society (2005).

Para Niederman (2005) o tubo e a VM reduzem o clearance da secreção das vias aéreas, favorecendo seu acúmulo no trato respiratório, aumentando os riscos. Outros fatores identificados foram: uso de antibióticos e bloqueador neuromuscular, reintubação, posição supina, sonda nasogástrica e transporte intra-hospitalar. 0 conhecimento dos fatores de risco é de fundamental importância para interferir na cadeia epidemiológica e na tomada de decisão do controle e prevenção da doença (AREVALO et al., 2011; ALMEIDA, POMBO, RODRIGUES, 2010).

Em uma pneumonia com incidência e impactos tão altos, medidas que visam à prevenção se mostram mais vantajosas que medidas de tratamento, relata Bonten (2011).

Por essa razão essas estratégias estão entre os temas mais estudados e debatidos entre os profissionais da UTI, seu controle e prevenção constituem um desafio e uma questão de importância econômica e social (AREVALO et al., 2011; BONTEN, 2011; ALMEIDA, POMBO, RODRIGUES, 2010; DEZFFULIAN et al., 2005).

As medidas de prevenção da PAVM podem ser divididas em farmacológicas e não farmacológicas, contudo estas tiveram destaque devido a sua maior viabilidade, eficácia e considerável menor custo. Essas estratégias já foram aderidas por várias instituições, contudo a sua adesão permanece um desafio, segundo Institute for Health Improvement (2008). A fisioterapia tem ampla e ativa participação na adoção e no gerenciamento de medidas não farmacológicas para a prevenção da PAVM, como:

A Fisioterapia Respiratória, incluindo procedimentos como drenagem postural, hiperinsuflação manual, percussões e vibrações torácicas, tem sido relatada como recurso para prevenir a PAVM. A combinação destas técnicas reexpandem atelectasias, favorecendo a melhora da complacência 
pulmonar, o fluxo expiratório e o clearance das secreções brônquicas, relatam Pattanshetty e Gaude (2010). Para Ntoumenopoulos et al., (2002) demonstram os seus benefícios na prevenção da patologia e indicam que a fisioterapia respiratória aplicada duas vezes ao dia sugere redução no risco de infecção e consequente diminuição na ocorrência da doença.

O uso dos Sistemas de Umidificação e Aerossolterapia, segundo Branson (2007) o fisioterapeuta exerce função fundamental no gerenciamento e uso adequado e otimizado de dispositivos associados ao uso de VM, como circuitos, filtros de umidificação, dispositivos de aerossolterapia e sistemas de aspiração. Os circuitos de ventiladores devem ser trocados quando há evidência de contaminação visível macroscopicamente com secreção do paciente.

Contudo, segundo Han e Liu (2010), sabe-se que os componentes do circuito são frequentemente contaminados durante as intervenções no paciente evidenciando que essa contaminação possa ter influência no desenvolvimento da PAVM.

A umidificação pode ser ativa, através de reservatórios de água no circuito, ou passiva, através de um dispositivo conhecido como filtros trocadores de calor e umidade (FTCU) que retém o calor e umidade exalada pelo paciente na expiração e devolve esse calor e umidade na inspiração. Estudos demonstraram que o tipo de umidificação não teve efeitos sobre complicações respiratórias do paciente. Há relatos do uso seguro do FTCU por até 3 a 7 dias, uma estratégia para o sucesso dessa prática e manter o filtro sempre acima da via aérea do paciente (RICARD, 2006; BRANSON, 2007).

Os nebulizadores são dispositivos acoplados ao circuito para administrar por via tópica fármacos, como corticoides e broncodilatadores, estes frequentemente são contaminados e podem contribuir para o desenvolvimento da PAVM. O uso de nebulímetros dosimetrados pode eliminar o risco de contaminação, além de ser de manuseio fácil e seguro, mais eficiente, com custo menor (BRANSON, 2007).

A utilização de Sistemas de Aspiração de Vias Aéreas para evitar o acúmulo de fluidos, que contêm bactérias residentes na orofaringe e estômago, acima do cuff pode diminuir o risco de aspiração, e assim as PAVM. Em uma meta-análise de 13 estudos, realizada por Bonten (2011), indicaram que essa prática foi associada com uma redução de $45 \%$ na PAVM, bem como na redução do tempo de permanência na UTI e do tempo de uso de VM.

Essa prática pode ser feita com tubos feitos especialmente para aspirar continuamente o local, esses diminuem a colonização bacteriana e consequentemente a PAVM, contudo podem promover lesões graves no local da aspiração, o que é agravado pela falta de sistemas de vácuos seguros na maioria dos hospitais, além do seu alto custo que inviabiliza seu uso (BONTEN, 2011; FERRER et al., 2009).

A Monitorização Rigorosa da Pressão do Cuff para a American Thoracic Society (2005), realizada de duas a três vezes ao dia em todos os pacientes com via aérea artificial, é uma medida fundamental para minimizar as microaspirações de secreções subglóticas, podendo reduzir o risco de PAVM, além de prevenir complicações causadas pela hiperinsuflação do balonete, como a estenose traqueal, traqueomalácea e fístulas gastroesofágicas, acrescenta Muscedere et al., (2008).

As estratégias de Higiene da Cavidade Oral, segundo estudos minimizam a colonização orofaríngea foram realizados, como o uso de antissépticos na higiene oral, entre os produtos utilizados está a clorexidina, um agente antimicrobiano com um largo espectro de atividade contra microorganismos gram-positivos (incluindo os resistentes) e com menor eficácia contra bacilos gram-negativos, segundo Andrade e Beraldo (2008). 
O produto é absorvido pelos tecidos e mantém sua atividade mesmo após cinco horas de administração. Outra vantagem do uso é que seu procedimento é seguro e bem tolerável, uma vez que não é conhecido qualquer efeito secundário. Além de ser considerada uma medida de baixo custo para o hospital comparado aos custos do tratamento de uma infecção (ANDRADE, BERALDO, 2008; BONTEN, 2011).

O Posicionamento no Leito para o Institue for Health Care Improvement (2008) manter a cabeceira elevada em 30 e $45^{\circ}$ em pacientes com nível de consciência baixo, com VM e dieta enteral é uma recomendação importante para a prevenção de PAVM.

Embora essa conduta, de prática simples, tenha demonstrado inúmeros benefícios sua adesão ainda é pequena. Essa posição favorece a melhor adaptação a VM, melhora a troca gasosa, diminui o risco de atelectasias e maior facilidade no desmame, além de favorecer o sistema cardiovascular, prevenir hipotensão postural e melhorar o estado de alerta do paciente (GOSSELINK et al., 2008; MUSCEDERE et al., 2008).

A implementação de Protocolos de Ventilação Mecânica Não Invasiva (VMNI) constituem uma conduta para diminuir a PAVM, uma vez que ela evita intubações e assim os riscos inerentes ao procedimento, segundo Hess (2005), considerando que um estudo sugeriu que o nome mais adequado seria "Pneumonia associada ao tubo endotraqueal".

Além disso, a VMNI ela também é usada no desmame de ventilação, colaborando para a redução do tempo de VM e os episódios de reintubação. Lembrando que o desenvolvimento da patologia está relacionado com a presença do tubo e não com a exposição ao ventilador (FERRER et al., 2009; HESS, 2005).

Os Programas de Educação permanente da Equipe Multiprofissional estão entre principais recomendações para reduzir a PAVM. Os Programas devem incluir a educação dos profissionais de saúde, a vigilância epidemiológica das infecções hospitalares, a interrupção na transmissão de microorganismos pelo uso apropriado de equipamento hospitalar, a prevenção da transmissão de uma pessoa para outra e a modificação dos fatores de riscos para o desenvolvimento de infecções bacterianas (ALMEIDA, POMBO, RODRIGUES, 2010).

A formação de equipes multiprofissionais comprometidas com o processo de educação permanente de programas de controle de infecções hospitalares pode resultar em um impacto econômico com diminuição dos custos hospitalares, afirmam Almeida, Pombo e Rodrigues (2010).

A implementação de Protocolos de Suspensão diária de Sedação, Desmame e Mobilização Precoce, pois quanto maior o tempo de VM, maior o risco de desenvolvimento da PAVM, diante disso estratégias para acelerar o processo de desmame é muito recomendado, associada a esse processo deve ser realizadas as interrupções da sedação para o fisioterapeuta avaliar a possibilidade de respiração espontânea e evoluir com o desmame, a sedação deve ser reiniciada quando há riscos para o paciente, como a assincronia ventilatória, agitação psicomotora e extubação acidental (ALMEIDA, POMBO, RODRIGUES, 2010; BONTEN, 2011).

Gosselink et al., (2008) relatam que com a interrupção da sedação o fisioterapeuta pode interagir com o paciente e realizar a avaliação neurofuncional e musculoesquelética, além de progredir com as mobilizações precoces, para melhorar a força muscular periférica e respiratória do paciente e ainda favorecer a funcionalidade e consequente qualidade de vida na pós alta da UTI, esses programas vem sendo muito usados para manter e recuperar a função e autonomia ventilatória do paciente, e assim, indiretamente, diminuir a PAVM. 


\section{OBJETIVOS}

Revisar as estratégias fisioterapêuticas na prevenção da PAVM, identificar o impacto e a importância da adoção de medidas de prevenção de fácil adesão, eficientes e de baixo custo além de descrever as principais estratégias fisioterapêuticas na adoção, gerenciamento e implantação de medidas para prevenção da PAVM.

\section{MATERIAIS E MÉTODOS}

Foi realizada pesquisa nas bases de dados Medline, IBECS, Cochrane, Lilacs e Scielo. Os artigos foram escolhidos através de seus conteúdos, devendo estar relacionados ao tema proposto neste estudo e nível de evidência A e/ou B nacional e internacional, utilizando os descritores Pneumonia, Respiração artifıcial. Serviço Hospitalar de Fisioterapia. Prevenção e controle.

Foram selecionados 24 estudos com objetivo de identificar os estudos de evidência científica, que abordam as mais relevantes prevalências da prevenção da pneumonia associada à ventilação mecânica (PAVM).

A pesquisa bibliográfica incluiu artigos originais, artigos de revisão, editoriais e diretrizes escritos nas línguas inglesa e portuguesa, sendo selecionados artigos publicados no período entre 2000 e 2014 .

\section{RESULTADOS E DISCUSSÃO}

A PAVM é responsável pelo alto índice de morbimortalidade dos pacientes internados em UTI e é um desafio para os intensivistas prevenir e controlar a doença (ALMEIDA, POMBO, RODRIGUES, 2010).

Avaliar o conhecimento dos profissionais de saúde nas UTI sobre prevenção de PAVM não é tarefa fácil, devido a muitas variáveis que estão envolvidas no processo de entendimento e ensinamento. Porém, a constante busca do conhecimento para uma assistência com mais qualidade é de primordial importância (AZEVEDO, 2006).

A maioria dos estudos relatados na literatura avaliando prevenção de PAVM considerou o efeito de apenas uma intervenção em reduzir a incidência dessa infecção. 0 que o presente estudo propôs foi uma abordagem multinível, agrupando medidas de prevenção simples e de baixíssimo custo.

A incidência de PAVM mostrou em diversos estudos uma tendência importante no declínio após a implantação de protocolos de profilaxia, com significado estatístico satisfatório (LUNA et al., 2006).

Dos estudos mencionados, dois que não encontraram risco aumentado de morte associado à pneumonia, um não arrolou pacientes clínicos e o outro só incluiu $26 \%$ desta categoria.

Ao contrário, dos três que apontaram aumento de mortalidade, um tinha $44 \%$ de pacientes clínicos e o outro $63 \%$. Neste último, a mortalidade absoluta observada foi $30,8 \%$ nos pacientes clínicos versus $12,1 \%$ nos cirúrgicos $(p=0,005)$, enquanto a mortalidade atribuível foi $65 \%$ versus $27,3 \%(p=0,04)$ em análise de sensibilidade (AZEVEDO, 2006).

Em uma análise multivariada, os pacientes críticos foram entubados e permaneceram em ventilação mecânica por pelo menos 48 horas, receberam intervenção fisioterapêutica, inclusa de drenagem 
postural, técnicas manuais respiratórias e aspiração das vias aéreas, duas vezes por dia, no qual se obteve resultados positivos e significativos na redução de PAVM (PATTANSHETTY, GAUDE, 2010).

Ntoumenopoulos et al., (2002) com resultados relevantes em seu estudo também preconiza a intervenção fisioterapêutica duas vezes ao dia como importante medida profilaxia indispensável.

Ensaios bem controlados têm sugerido que a drenagem postural ou a contínua aspiração supra glótica pode reduzir consideravelmente o risco de PAVM (GUIMARÃES, ROCCO, 2006; FAGON, 2000).

No entanto, uma revisão sistemática, com base em pós-operatórios sugeriu que a fisioterapia respiratória não traz benefícios na prevenção de PAVM (KIRTON, 2000). Contudo, a atual pesquisa obteve um contraste, onde sugere que o uso da fisioterapia respiratória combinada pode ser útil e de suma importância em pacientes críticos na prevenção de PAVM.

Outras tentativas não-farmacológicas para reduzir PAVM incluiu a utilização de permutadores de calor e de umidade reduzindo alterações nos circuitos dos ventiladores (GUIMARÃES, ROCCO, 2006; FAGON, 2000; KIRTON, 2000; CRAVEN, HJALMARSON, 2010).

Com base nos estudos analisados (em todos a clorexidina foi administrada periodicamente, usando uma técnica padrão, durante o período em que o paciente permaneceu em VM), foi concluído que o uso tópico de clorexidina na higiene bucal de pacientes sob ventilação mecânica parece diminuir a colonização da cavidade oral, reduzindo assim a incidência da patologia. Contudo novos estudos são necessários para determinar sua concentração ideal, bem como a forma mais adequada de apresentação, frequência e técnica de administração (ANDRADE, BERALDO, 2008).

A fisioterapia pode ter participação ampla e ativa na adoção e gerenciamento de medidas não farmacológicas para a prevenção da PAVM, autores afirmam que ainda é necessário um maior número de pesquisas para concluir o benéfico da fisioterapia na prevenção da patologia (BRANSON, 2005; HAN, LIU, 2010; HESS, 2005; MUSCEDERE et al., 2008).

Estudos adicionais sob a forma da intervenção clínica em pacientes com risco de PAVM pode confirmar estes resultados e estabelecer qual a combinação de técnicas fisioterapêuticas têm um potencial mais relevante na prevenção de PAVM.

\section{CONSIDERAÇÕES FINAIS}

Com alta incidência e com um impacto significativo na morbidade, mortalidade, tempo de internação e consequentemente custo do paciente para a instituição a prevenção da PAVM é fundamental, sobretudo com medidas não farmacológicas, que se mostram mais viáveis e menos onerosas. Essas estratégias simples apresentam evidências científicas suficientes quanto seus benefícios e resultados positivos na redução do risco da patologia, contudo a falta de adesão na prática clínica contribui para sua incidência tão expressiva.

Torna-se clara a importância do fisioterapeuta no atendimento desses pacientes com sua participação ampla e ativa na equipe multiprofissional, no processo de planejamento, implantação e gerenciamento de medidas não farmacológicas para a prevenção da PAVM. Contudo ainda são necessários mais estudos e pesquisas para melhorar as evidências científicas sobre o assunto. 


\section{REFERÊNCIAS}

AKÇA O.; KOLTKA K.; UZEL S.; ÇAKAR N. Risk factors for early onset ventilator associated pneumonia in critical care patients. Anesthesiol, n. 93, p. $638-645,2000$.

ALMEIDA PC; POMBO CN; RODRIGUES JL. Conhecimento dos profissionais de saúde na Unidade de Terapia Intensiva sobre prevenção de pneumonia associada à ventilação mecânica. Ciênc. saúde coletiva, n. 1, v. 15, 2010.

AMERICAN Thoracic Society. Infectious Diseases Society of America. Guidelines for the management of adults with hospital-acquired, ventilator-associated, and healthcare associated pneumonia. Am J Respire Crit Care Med, n. 4, v. 171, p. 388 - 416, 2005.

ANDRADE D.; BERALDO C. Oral hygiene with chlorhexidine in preventing pneumonia associated with mechanical ventilation. J Bras Pneumol, n. 9, v. 34, p. 707-14, 2008.

AREVALO NL; ROLANDO RS; IVAN A. FUENTES P. Pneumonia associated with mechanical ventilation in the Intensive Care Unit. Comité de IIH Unidad de Cuidados Intensivos Universidad Católica del Norte do Chile, n. 4, v. 28, p. 316-332, 2011.

AZEVEDO F. Pneumonia associada à ventilação mecânica: análise de fatores epidemiológicos na confecção de estratégias de profilaxia e terapêutica. Rev. Bras. Ter. Intensiva, n. 4, v. 18, 2006.

BONTEN M. Ventilator-associated Pneumonia and the Gastropulmonary Route of Infection. Am. J. Resp. and Critical Care Med, n. 9, v. 184, p. 991-993, 2011.

BRANSON RD. Secretion management in the mechanically ventiled patient. Respir Care, n. 10, v. 52, p. 328-342, 2007.

BRANSON RD. The ventilator circuit and ventilator-associated pneumonia. Respir Care, n. 6, v. 50, p. 774-785, 2005.

CRAVEN D.; HJALMARSON K. Ventilator-Associated Tracheobronchitis and Pneumonia: Thinking Outside the Box. Clinical Infectious Diseases, n. 5, v. 77, 2010.

DEZFFULIAN C.; SHOJANIA K.; COLLARD HR.; KIM HM.; MATTHAY MA; SAINT S. Subglottic secretion drainage for preventing ventilator-associated pneumonia: a metaanayisis. Am J Med, n. 1, v. 118, 2005.

FAGON JY.; CHASTRE J.; WOLFF M.; GERVAIS C.; PARER-AUBAS S.; STEPHAN F.; SIMILOWSKI T.; MERCAT A.; DIEHL JL.; SOLLET JP.; TENAILLON A. Invasive and noninvasive strategies for management of suspected ventilatorassociated pneumonia. A randomised trial. Ann Intern Med, n, 132, p. 621-630, 2000.

FERRER M.; SELLARES J.; VALENCIA M.; CARRILO A.; GONZALEZ G.; BADIA JR.; NICOLAS JM.; TORRES, A. xTorres Non-inavisive ventilation after extubation in hypercapnic patients with cronic respiratory dosorders: randomised controlled trial. Lancet, n. 364, 2009.

GOSSELINK R.; BOOT J.; JHONSON M.; DEAN E.; NAVA S.; NORREMBERG M, SCHÖNHOFER B.; STILLER K.; VAN DE LEUR H.; VINCENT JL. Physioterapy for adult patients with critical illness: recommendations oh the European Respiratory Society and European Society of Intensive Care Medicine Task Force on Physiotherapy for Critically III Patients. Intensive Care Med, n. 7, v. 34, 2008. 
GUIMARÃES MMQ.; ROCCO JR. Prevalência e prognóstico dos pacientes com pneumonia associada á ventilação mecânica em um hospital universitário. J Bras Pneumo, n. 4, v.32, 2006.

HAN J.; LIU Y. Effect of ventilator circuite changes on ventilator-associated pneumonia: a systematic review and meta-analysis. Respir Care, n. 4, v. 55, 2010.

HESS D. Noinvasive positive-pressure ventilation and ventilator-associated pneumonia. Respir Care, n. 7, v. 50, p. 924-929, 2008.

KIRTON OC.; DEHAVEN B.; MORGAN J.; MOREJON O.; CIVETTA J. A prospective randomised comparison of an in-line heat moisture exchange filter and heated wire humdifiers: rates of ventilator-associated early-onset (community- acquired) or lateonset (hospital- acquired) pneumonia and incidence of endotracheal tube occlusion. Chest, n. 112, 2000.

LUNA CM.; ARUJ P.; NIEDERMAN MS.; GARZON J.; VIOLI D.; PRIGNONI A.; RÍOS F.; BAQUERO S.; GANDO $\mathrm{S}$. Appropriateness and delay to initiate therapy in ventilator-associated pneumonia. Eur Respir $\mathrm{J}, \mathrm{n}$. 1, v. 27, 2006.

MUSCEDERE J.; DODEK P.; KEENAN S.; FOWLER R.; COOK D.; HEYLAND D. Comprehensive evidencebased clinical practice guidelines for ventilator-associated pineumonia: prevention. J Crit Care, $n .1, v$. 23, p. 126-37, 2008.

NIEDERMAN MS. The clinical diagnosis of ventilator-associated pneumonia. Respir Care, n. 5, v. 50, $p$. 788-796, 2005.

NTOUMENOPOULOS G.; PRESNEILL J.; MCELHOLUM CADE J. Chest physiotherapy for the prevention of ventilator-associeted pneumonia. Intensive Care, n.7, v. 28, 2002.

PATTANSHETTY R.; GAUDE G. Efect of multimodality chest physiotherapy in the prevention of ventilatorassocieted pneumonia: A randomized clinical trial. Indian J Crit Care Med, n. 2, v. 14, p. 70-76, 2010.

RICARD J. Humidification. In: Tobin M, editor. Mechanical ventilation. New York: McGraw-Hill, p. 1109$1120,2006$. 